\title{
Generalized Ordered Weighted Simplified Neutrosophic Cosine Similarity Measure for Multiple Attribute Group Decision Making
}

\author{
Jun Ye, Shaoxing University, Shaoxing, China
}

\begin{abstract}
The paper proposes a generalized ordered weighted simplified neutrosophic cosine similarity (GOWSNCS) measure by combining the cosine similarity measure of simplified neutrosophic sets (SNSs) with the generalized ordered weighted averaging (GOWA) operator and investigates its properties and special cases. Then, the author develops a simplified neutrosophic group decisionmaking method based on the GOWSNCS measure to handle multiple attribute group decision-making problems with simplified neutrosophic information. The prominent characteristics of the GOWSNCS measure are that it not only is a generalization of the cosine similarity measure but also considers the associated weights for attributes and decision makers in the aggregation of the cosine similarity measures of SNSs to alleviate the influence of unduly large or small similarities in the process of information aggregation. Finally, an illustrative example of investment alternatives is provided to demonstrate the application and effectiveness of the developed approach.
\end{abstract}

\section{KEYWORDS}

Cosine Similarity Measure, Generalized Ordered Weighted Simplified Neutrosophic Cosine Similarity (GOWSNCS) Measure, Group Decision Making, Simplified Neutrosophic Set

\section{INTRODUCTION}

In complex engineering, economics, and management, multiple attribute group decision making is a very important research topic (Zheng et al. 2018, Lin et al. 2018, Liu et al. 2019a and 2019b). Although fuzzy sets (Zadeh 1965), intuitionistic fuzzy sets (IFSs) (Atanassov 1986), and intervalvalued intuitionistic fuzzy sets (IVIFSs) (Atanassov and Gargov 1989) have been developed in vague, incomplete, and uncertain setting, they cannot describe and deal with indeterminate and inconsistent information in various real problems. In this case, Smarandache (1999) proposed the concept of a neutrosophic set as a generalization of the concepts of the classic set, fuzzy set, IFS and IVIFS. In the neutrosophic set, a truth-membership $T(x)$, an indeterminacy-membership $I(x)$ and a falsitymembership $F(x)$ are represented independently and lie within the real standard or nonstandard unit interval $]^{-} 0,1^{+}[$. Then, the indeterminacy presented in the neutrosophic set is independent on the truth and falsity values and can include inconsistent information, while the incorporated uncertainty in the IFS is dependent on the degrees of belongingness and non-belongingness and cannot include inconsistent information. Hence, the neutrosophic set can better express incomplete, indeterminate and inconsistent information. However, the neutrosophic set is difficult to be applied in real-life situations due to the nonstandard unit interval $]^{-} 0,1^{+}[$for the range of the three functions $T(x), I(x)$ and $F(x)$. Thus, the range of the functions $T(x), I(x)$ and $F(x)$ can be restrained to the real standard unit interval 
$[0,1]$ to be easily applied in real science and engineering problems. Consequently, a single-valued neutrosophic set (SVNS) (Wang et al. 2010), an interval neutrosophic set (INS) (Wang et al. 2005), and a simplified neutrosophic set (SNS) (Ye 2014a) were introduced by some researchers. Then, SNSs are the subclass of neutrosophic sets (Ye 2014a) and include the concepts of SVNSs and INSs.

In recent years, SNSs have been mainly applied to decision making and medical diagnosis problems. Ye (2013) presented a correlation coefficient of SVNSs and applied it to single-valued neutrosophic multiple attribute decision-making problems. Then, Ye (2014b) introduced the crossentropy measure of SVNSs and its single-valued neutrosophic multiple attribute decision-making method. Further, Ye (2014c) put forward the distances-based similarity measures of INSs and their interval neutrosophic multicriteria decision-making method. Moreover, Chi and Liu (2013) proposed an extended TOPSIS method for interval neutrosophic multiple attribute decision making problems. Peng et al. (2014) introduced an outranking approach for multicriteria decision-making problems with simplified neutrosophic information. Zhang et al. (2014) developed the interval neutrosophic number weighted average (INNWA) and interval neutrosophic number weighted geometric (INNWG) operators and their interval neutrosophic multiple attribute decision-making method. Liu and Wang (2014) also developed single-valued neutrosophic normalized weighted Bonferroni mean operators for single-valued neutrosophic decision-making problems. Further, Liu et al. (2014) developed some generalized single-valued neutrosophic number Hamacher aggregation operators and their singlevalued neutrosophic group decision-making method. Also, Ye (2014d) developed a multiple attribute group decision-making method with completely unknown weights based on similarity measures under single-valued neutrosophic environment. Ye (2014e) presented vector similarity measures of SNSs and their application in multicriteria decision making. Ye (2015) further proposed the improved cosine similarity measures of SNSs based on the cosine function to overcome some disadvantages of the cosine similarity measure of SNSs in vector space and applied them to medical diagnoses. Zhang et al. (2016) also introduced an outranking approach for multicriteria decision-making problems with interval neutrosophic information. Furthermore, Yang and Li (2016) introduced power aggregation operators of single-valued neutrosophic sets and used them for decision making. Then, Sahin and Liu (2017) presented possibility-induced simplified neutrosophic aggregation operators for group decision-making problems. Ye (2017) put forward simplified neutrosophic harmonic averaging projection method and applied it to multiple attribute decision making problems. Further, Tu et al. (2018) proposed simplified neutrosophic symmetry measures for decision making Problems.

However, in the aforementioned decision making methods based on similarity measures, they scarcely consider the importance of the ordered position of each similarity degree. In some cases, the important degree of the ordered positions of arguments is very important in the process of information aggregation (Yager 2004; Zhou et al 2014). Hence, we may need to consider the weights of the ordered position of arguments in the information aggregation. For example, in the diving contest of Olympic Games, generally one can take the average value of the remaining scores after removing the highest and lowest scores, i.e., one can assign that the weights of the highest and lowest scores are 0 . Therefore, the positional weights are very important in some real group decision making problems. To alleviate the influence of unduly large or small similarities in the process of information aggregation, we should assign them specific weights. In such situations, the degrees of similarity can be rearranged in descending order, and then aggregated together with the weights of their ordered positions. Obviously, the existing decision-making methods based on similarity measures are unsuitable for dealing with such cases under simplified neutrosophic environment. Thus, we need to define ordered weighted aggregation operators for the cosine similarity measure of SNSs to handle the decision-making problems. Motivated by the generalized ordered weighted averaging (GOWA) operator (Yager 2004) and the intuitionistic fuzzy ordered weighted cosine similarity measure (Zhou et al 2014), the purposes of this paper are to develop a generalized ordered weighted simplified neutrosophic cosine similarity (GOWSNCS) measure by combining the simplified neutrosophic cosine similarity measure with the GOWA operator as an extension of the two major works in (Yager 2004 and Zhou et al 2014) and its group decision making method in SNS setting. It is obvious that 
the proposed decision making method can alleviate the main problem gap between unduly large and small similarities and show the GOWA flexibility by taking possible values of the parameter $\lambda$ in the decision making process, which shows its main advantages.

The rest of the paper is organized as follows. Section 2 briefly describes some basic concepts of SNSs, the cosine similarity measure of SNSs, and the GOWA operator. In Section 3, we propose the GOWSNCS measure by combining the simplified neutrosophic cosine similarity measure with the GOWA operator and investigate their properties and special cases. Section 4 establishes a multiple attribute group decision-making method based on the GOWSNCS measure under simplified neutrosophic environment. An illustrative example is provided to demonstrate the application of the proposed group decision-making method in Section 5. Section 6 gives conclusions and future research.

\section{PRELIMINARIES}

This section briefly reviews SNSs, the cosine similarity measure of SNSs, and the GOWA operator.

\subsection{Some Basic Concepts of SNSs and the Cosine Similarity Measure}

To apply a neutrosophic set to science and engineering areas, Ye (2014a) introduced the SNS concept, which is a subclass of the neutrosophic set, and gave the following definition of a SNS.

Definition 1 (Ye 2014a): Let $X$ be a space of points (objects), with a generic element in $X$ denoted by $x$. A neutrosophic set $B$ in $X$ is characterized by a truth-membership function $T_{B}(x)$, an indeterminacymembership function $I_{B}(x)$, and a falsity-membership function $F_{B}(x)$. If the functions $T_{B}(x), I_{B}(x)$ and $F_{B}(x)$ are singleton subintervals/subsets in the real standard $[0,1]$, such that $T_{B}(x): X \rightarrow[0$, $1], I_{B}(x): X \rightarrow[0,1]$, and $F_{B}(x): X \rightarrow[0,1]$. Then, a SNS $B$ is denoted by:

$$
B=\left\{\left\langle x, T_{B}(x), I_{B}(x), F_{B}(x)\right\rangle \mid x \in X\right\}
$$

The SNS is a subclass of the neutrosophic set and includes the concepts of INS and SVNS (Ye 2014a). For convenience, the triple $\left\langle T_{B}(x), I_{B}(x), F_{B}(x)\right\rangle$ in the SNS $B$ is a basic element denoted by $b$ $=\langle T, I, F\rangle$, which is called a simplified neutrosophic value (SNV). In this paper, we only consider $T$, $I, F \in[0,1]$ as three real numbers in the real unit interval $[0,1]$ and their sum satisfies the condition $0 \leq T+I+F \leq 3$.

Let $b_{1}=\left\langle T_{1}, I_{1}, F_{1}\right\rangle$ and $b_{2}=\left\langle T_{2}, I_{2}, F_{2}\right\rangle$ be two SNVs in $X$. Then there are the following relations (Ye 2014a):

1. Complement: $b_{1}{ }^{c}=\left\langle F_{1}, 1-I_{1}, T_{1}\right\rangle$;

2. Inclusion: $b_{1} \subseteq b_{2}$ if and only if $T_{1} \leq T_{2}, I_{1} \geq I_{2}, F_{1} \geq F_{2}$;

3. Equality: $b_{1}=b_{2}$ if and only if $b_{1} \subseteq b_{2}$ and $b_{2} \subseteq b_{1}$.

For two SNVs $b_{1}=\left\langle T_{1}, I_{1}, F_{1}\right\rangle$ and $b_{2}=\left\langle T_{2}, I_{2}, F_{2}\right\rangle$, based on the improved cosine similarity measure of SNSs proposed by Ye $(2014 \mathrm{e})$, we introduce the following cosine similarity measure between $b_{1}$ and $b_{2}$ :

$$
C\left(b_{1}, b_{2}\right)=\cos \left[\frac{\pi\left(\left|T_{1}-T_{2}\right|+\left|I_{1}-I_{2}\right|+\left|F_{1}-F_{2}\right|\right)}{6}\right]
$$


Then, the cosine similarity measure $C\left(b_{1}, b_{2}\right)$ satisfies the following properties (Ye 2014e):

P1: $0 \leq C\left(b_{1}, b_{2}\right) \leq 1$

P2: $C\left(b_{1}, b_{2}\right)=1$ if and only if $b_{1}=b_{2}$

P3: $C\left(b_{1}, b_{2}\right)=C\left(b_{2}, b_{1}\right)$

P4: If $b_{3}$ is a SNV and $b_{1} \subseteq b_{2} \subseteq b_{3}$, then $C\left(b_{1}, b_{3}\right) \leq C\left(b_{1}, b_{2}\right)$ and $C\left(b_{1}, b_{3}\right) \leq C\left(b_{2}, b_{3}\right)$

\subsection{GOWA Operator}

Yager (2004) proposed the GOWA operator to provide a parameterized family of aggregation operators between the minimum and the maximum and gave the definition of the GOWA operator.

Definition 2 (Yager 2004): The GOWA operator of $n$ dimensions is a mapping GOWA: $R^{n} \rightarrow R$, defined by the following formula:

$\operatorname{GOWA}\left(a_{1}, a_{2}, \ldots, a_{n}\right)=\left\{\sum_{j=1}^{n} w_{j}\left(a_{\sigma(j)}\right)^{\lambda}\right\}^{1 / \lambda}$

where $w=\left(w_{1}, w_{2}, \ldots, w_{n}\right)^{\mathrm{T}}$ is an associated weight vector with $w_{j} \in[0,1]$ and $\sum_{j=1}^{n} w_{j}=1,(\sigma(1)$, $\sigma(2), \ldots, \sigma(n))$ is a permutation of $(1,2, \ldots, n)$ such that $a_{\sigma(j-1)} \geq a_{\sigma(j)}$ for $j=2,3, \ldots, n$ in the argument $a_{j}(j=1,2, \ldots, n)$, and a parameter $\lambda \in(-\infty, \infty)$ and $\lambda \neq 0$.

The GOWA operator is monotonic, commutative, bounded and idempotent (Yager 2004). Taking possible values of the parameter $\lambda$, one can obtain a group of particular cases. For example, if $\lambda=1$, the GOWA operator becomes the OWA operator; if $\lambda \rightarrow 0$, the GOWA operator becomes the ordered weighted geometric averaging (OWGA) operator; if $\lambda=-1$, the GOWA operator becomes the ordered weighted harmonic averaging (OWHA) operator.

\section{GENERALIZED ORDERED WEIGHTED SIMPLIFIED NEUTROSOPHIC COSINE SIMILARITY MEASURE}

This section proposes a GOWSNCS measure by combining the cosine similarity measure of SNVs with the GOWA operator.

Let $A=\left\{a_{1}, a_{2}, \ldots, a_{n}\right\}$ and $B=\left\{b_{1}, b_{2}, \ldots, b_{n}\right\}$ be two SNSs, where $a_{j}=\left\langle T_{a j}, I_{a j}, F_{a j}\right\rangle$ and $b_{j}=$ $\left\langle T_{b j}, I_{b j}, F_{b j}\right\rangle(j=1,2, \ldots, n)$ are SNVs, $T_{a j}, I_{a j}, F_{a j} \in[0,1]$ and $T_{b j}, I_{b j}, F_{b j} \in[0,1]$. Then we can define the GOWSNCS measure as follows:

Definition 3: For two SNSs $A$ and $B$, the GOWSNCS measure of $A$ and $B$ is a mapping GOWSNCS: $R^{n} \rightarrow R$, which is defined as:

$\operatorname{GOWSNCS}(A, B)=\left\{\sum_{j=1}^{n} w_{j}\left[C\left(a_{\sigma(j)}, b_{\sigma(j)}\right)\right]^{\lambda}\right\}^{1 / \lambda}$

where $w=\left(w_{1}, w_{2}, \ldots, w_{n}\right)^{\mathrm{T}}$ is an associated weight vector with $w_{j} \in[0,1], \sum_{j=1}^{n} w_{j}=1,(\sigma(1), \sigma(2)$, $\ldots, \sigma(n))$ is a permutation of $(1,2, \ldots, n)$, such that $C\left(a_{\sigma(j-1)}, b_{\sigma(j-1)}\right) \geq C\left(a_{\sigma(j)}, b_{\sigma(j)}\right)$ for $j=2,3, \ldots, n$, 
$C\left(a_{\sigma(j)}, b_{\sigma(j)}\right)$ is the cosine similarity measure between $a_{j}$ and $b_{j}(j=1,2, \ldots, n)$ for the parameter $\lambda>$ 0 .

Then, the GOWSNCS measure is monotonic, idempotent, bounded, commutative, nonnegative, and reflexive. These desirable properties are as follows:

1. Monotonicity: For three SNSs $A=\left\{a_{1}, a_{2}, \ldots, a_{n}\right\}, B=\left\{b_{1}, b_{2}, \ldots, b_{n}\right\}$ and $C=\left\{c_{1}, c_{2}, \ldots, c_{n}\right\}$, If $C\left(a_{j}, b_{j}\right) \leq C\left(a_{j}, c_{j}\right)$ for $j=1,2, \ldots, n$, then $\operatorname{GOWSNCS}(A, B) \leq \operatorname{GOWSNCS}(A, C)$;

2. Idempotency: For two SNSs $A=\left\{a_{1}, a_{2}, \ldots, a_{n}\right\}$ and $B=\left\{b_{1}, b_{2}, \ldots, b_{n}\right\}$, if all the cosine measures of $C\left(a_{j}, b_{j}\right)(j=1,2, \ldots, n)$ are equal, i.e., $\mathrm{C}\left(a_{j}, b_{j}\right)=V$ for $j=1,2, \ldots, n$, then $\operatorname{GOWSNCS}(A$, $B)=V$;

3. Boundedness: For two SNSs $A=\left\{a_{1}, a_{2}, \ldots, a_{n}\right\}$ and $B=\left\{b_{1}, b_{2}, \ldots, b_{n}\right\}$, if $\max _{j} C\left(a_{j}, b_{j}\right)=V_{\max }$ and $\min _{j} C\left(a_{j}, b_{j}\right)=V_{\min }$, then $V_{\min } \leq \operatorname{GOWSNCS}(A, B) \leq V_{\max }$;

4. Commutativity (GOWA aggregation): For two SNSs $A=\left\{a_{1}, a_{2}, \ldots, a_{n}\right\}$ and $B=\left\{b_{1}, b_{2}, \ldots\right.$, $\left.b_{n}\right\}$, if $\left(C\left(a_{1}, b_{1}\right), C\left(a_{2}{ }^{\prime}, b_{2}{ }^{\prime}\right), \ldots, C\left(a_{n}, b_{n}{ }^{\prime}\right)\right)$ is a permutation of $\left(C\left(a_{1}, b_{1}\right), C\left(a_{2}, b_{2}\right), \ldots, C\left(a_{n}\right.\right.$, $\left.b_{n}\right)$ ), then GOWSNCS $\left(A^{\prime}, B^{\prime}\right)=\operatorname{GOWSNCS}(A, B)$, where $A^{\prime}=\left\{a_{1}, a_{2}, \ldots, a_{n}^{\prime}\right\}$ and $B^{\prime}=\left\{b_{1}{ }^{\prime}\right.$, $\left.b_{2}, \ldots, b_{n}{ }^{\prime}\right\}$;

5. Commutativity (similarity measure): For two SNSs $A=\left\{a_{1}, a_{2}, \ldots, a_{n}\right\}$ and $B=\left\{b_{1}, b_{2}, \ldots\right.$, $\left.b_{n}\right\}$, there is $\operatorname{GOWSNCS}(A, B)=\operatorname{GOWSNCS}(B, A)$;

6. Nonnegativity: For two SNSs $A=\left\{a_{1}, a_{2}, \ldots, a_{n}\right\}$ and $B=\left\{b_{1}, b_{2}, \ldots, b_{n}\right\}$, there is $0 \leq$ $\operatorname{GOWSNCS}(A, B) \leq 1$;

7. Reflexivity: For a $\operatorname{SNS} A=\left\{a_{1}, a_{2}, \ldots, a_{n}\right\}$, there is $\operatorname{GOWSNCS}(A, A)=1$.

If we consider the possible values of the parameter $\lambda$ in the GOWSNCS measure, we can obtain a group of particular cases:

1. If $\lambda=1$, then the GOWSNCS measure reduces to the ordered weighted arithmetic simplified neutrosophic cosine similarity (OWASNCS) measure of $A$ and $B$ :

$O W A S N C S(A, B)=\sum_{j=1}^{n} w_{j} C\left(a_{\sigma(j)}, b_{\sigma(j)}\right)$

2. If $\lambda=2$, then the GOWSNCS measure becomes the ordered weighted quadratic simplified neutrosophic cosine similarity (OWQSNCS) measure of $A$ and $B$ :

$\operatorname{OWQSNCS}(A, B)=\left\{\sum_{j=1}^{n} w_{j}\left[C\left(a_{\sigma(j)}, b_{\sigma(j)}\right)\right]^{2}\right\}^{1 / 2}$

3. If $\lambda \rightarrow 0$, then the GOWSNCS measure becomes the ordered weighted geometric simplified neutrosophic cosine similarity (OWGSNCS) measure of $A$ and $B$ :

$O W G S N C S(A, B)=\prod_{j=1}^{n}\left[C\left(a_{\sigma(j)}, b_{\sigma(j)}\right)\right]^{w_{j}}$ 


\section{MULTIPLE ATTRIBUTE GROUP DECISION MAKING USING THE GOWSNCS MEASURE}

In this section, we develop a group decision making method to apply the GOWSNCS measure to multiple attribute group decision making problems with simplified neutrosophic information.

For a multiple attribute group decision-making problem, let $U=\left\{u_{1}, u_{2}, \ldots, u_{m}\right\}$ be a set of $m$ feasible alternatives and $G=\left\{g_{1}, g_{2}, \ldots, g_{n}\right\}$ be a set of $n$ attributes, and $E=\left\{e_{1}, e_{2}, \ldots, e_{t}\right\}$ be a set of $t$ decision makers. Assume that $w=\left(w_{1}, w_{2}, \ldots, w_{n}\right)^{\mathrm{T}}$ is an associated weight vector for the attributes with $w_{j} \in[0,1]$ and $\sum_{j=1}^{n} w_{j}=1$, and $\omega=\left(\omega_{1}, \omega_{2}, \ldots, \omega_{t}\right)^{\mathrm{T}}$ is an associated weight vector for the decision makers with $\omega_{k} \in[0,1]$ and $\sum_{k=1}^{t} \omega_{k}=1$. When a group of decision makers are required to evaluate the alternative $u_{i} \in U$ with respect to the attribute $g_{j} \in G$, each decision maker provides his/her own decision matrix $D^{(k)}=\left(d_{i j}^{(k)}\right)_{m \times n}$, where $d_{i j}^{(k)}=\left\langle T_{i j}^{(k)}, I_{i j}^{(k)}, F_{i j}^{(k)}\right\rangle(i=1,2, \ldots, m ; j=1$, $2, \ldots, n ; k=1,2, \ldots, t)$ is the SNV given by the decision maker $e_{k} \in E$.

The process of the multiple attribute group decision making method involves the following steps:

Step 1: Establish the ideal alternatives by giving the ideal level of each attribute for each decision maker, which is represented by the ideal neutrosophic value:

$d_{j}^{*(k)}=\left\langle T_{j}^{*(k)}, I_{j}^{*(k)}, F_{j}^{*(k)}\right\rangle=\left\langle\max _{i} T_{i j}^{(k)}, \min _{i} I_{i j}^{(k)}, \min _{i} F_{i j}^{(k)}\right\rangle$

for $i=1,2, \ldots, m$ and $j=1,2, \ldots, n$ in the ideal alternative $d^{*(k)}=\left\{d_{1}^{*(k)}, d_{2}^{*(k)}, \ldots, d_{n}^{*(k)}\right\}$ for $k=1$, $2, \ldots, t$.

Step 2: Calculate the collective cosine value of GOWSNCS $\left(d_{i}^{(k)}, d^{*(k)}\right)(i=1,2, \ldots, m ; k=1,2, \ldots$, $t$ ) by the following formula:

$V_{i}^{(k)}=G O W C S\left(d_{i}^{(k)}, d^{*(k)}\right)=\left\{\sum_{j=1}^{n} w_{j}\left[C\left(d_{i \sigma(j)}^{(k)}, d_{\sigma(j)}^{*(k)}\right)\right]^{\lambda}\right\}^{1 / \lambda}$

where $d_{i}^{(k)}=\left\{d_{i 1}^{(k)}, d_{i 2}^{(k)}, \ldots, d_{i n}^{(k)}\right\}$ and $d^{*(k)}=\left\{d_{1}^{*(k)}, d_{2}^{*(k)}, \ldots, d_{n}^{*(k)}\right\}$.

Step 3: Aggregate all the collective cosine values of $V_{i}^{(k)}(k=1,2, \ldots, t)$ into the collective overall value $R_{i}$ for the alternative $u_{i}(i=1,2, \ldots, m)$ by the following aggregation formula:

$R_{i}=\left\{\sum_{k=1}^{t} \omega_{k}\left(V_{i}^{\sigma(k)}\right)^{\lambda}\right\}^{1 / \lambda}$

where $(\sigma(1), \sigma(2), \ldots, \sigma(t))$ is a permutation of $(1,2, \ldots, t)$, such that $V_{i}^{\sigma(k-1)} \geq V_{i}^{\sigma(k)}$ for $k=2,3, \ldots$, $t$. 
Step 4: Rank the alternatives of $u_{i}(i=1,2, \ldots, m)$ in a descending order according to the collective overall value $R_{i}(i=1,2, \ldots, m)$ and select the best alternative in accordance with the ranking order of the alternatives.

Step 5: End.

\section{ILLUSTRATIVE EXAMPLE}

For convenient comparison, a numerical example discussed in Ye (2014f) is adapted to demonstrate the applications and effectiveness of the proposed method.

Assume that an investment company wants to invest a sum of money in the best option. There is a panel with five possible alternatives: (1) $u_{1}$ is a car company; (2) $u_{2}$ is a food company; (3) $u_{3}$ is a computer company; (4) $u_{4}$ is an arms company; (5) $u_{5}$ is a TV company. The investment company must make a decision according to the four attributes: (1) $g_{1}$ is the risk; (2) $g_{2}$ is the growth; (3) $g_{3}$ is the social political impact; (4) $g_{4}$ is the environmental impact. Then, the associated weight vector $w$ $=(0.22,0.3,0.26,0.22)^{\mathrm{T}}$ is given for the attributes in the GOWSNCS measure. Assume that three decision makers or experts for $E=\left\{e_{1}, e_{2}, e_{3}\right\}$ are required in the evaluation process and the associated weight vector for the three decision makers is $\omega=(0.3,0.4,0.3)^{\mathrm{T}}$.

Therefore, the five possible alternatives of $u_{i}(i=1,2,3,4,5)$ are evaluated by the three decision makers of $e_{k}(k=1,2,3)$ under the four attributes of $g_{j}(j=1,2,3,4)$ on the fuzzy concept "excellence" and the evaluation values are represented by the form of SNVs. Therefore, the three simplified neutrosophic decision matrices can be given by the three decision makers, which are expressed, respectively, as follows (Ye 2014f):

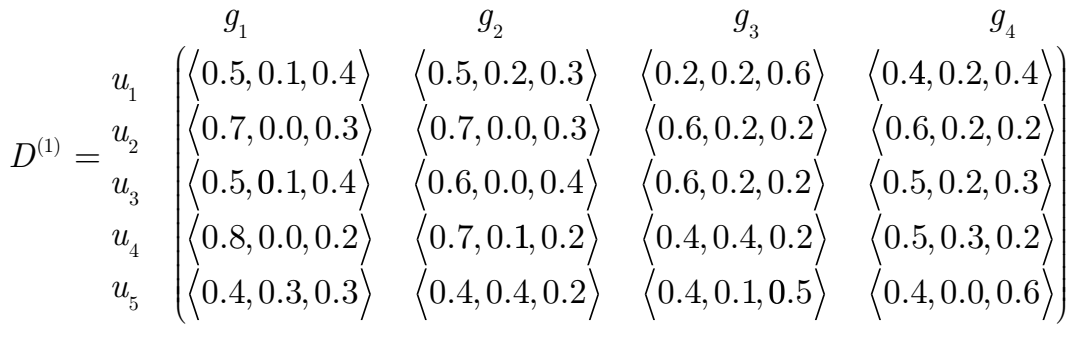

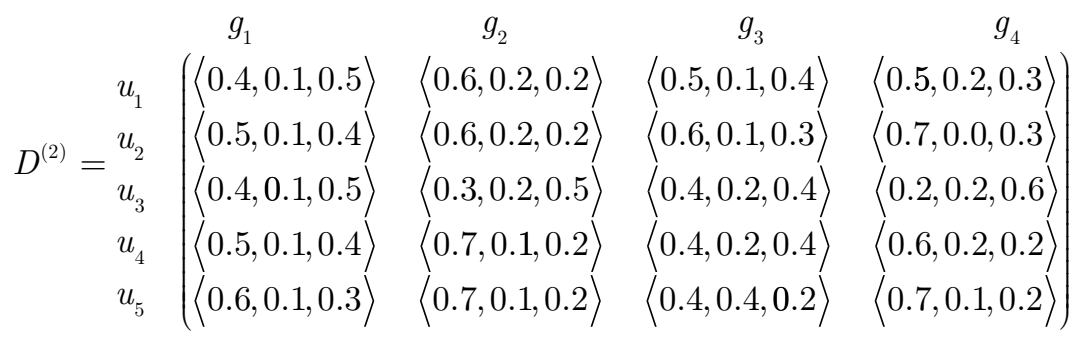

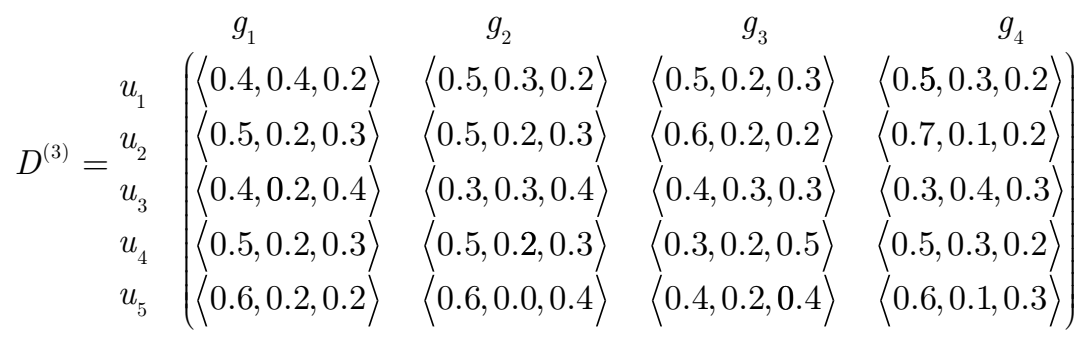


Thus, we use the proposed group decision making method to rank the alternatives and obtain the most desirable one(s). Let us take $\lambda=1$ for demonstrating the computing procedure of the proposed method:

Step 1: According to the ideal simplified neutrosophic value:

$$
d_{j}^{*(k)}=\left\langle T_{j}^{*(k)}, I_{j}^{*(k)}, F_{j}^{*(k)}\right\rangle=\left\langle\max _{i} T_{i j}^{(k)}, \min _{i} I_{i j}^{(k)}, \min _{i} F_{i j}^{(k)}\right\rangle
$$

for $i=1,2,3,4,5 ; j=1,2,3,4$ and $k=1,2,3$, we can yield the three ideal alternatives from the above three simplified neutrosophic decision matrices given by the three decision makers, respectively, as follows:

$$
\begin{aligned}
& d^{*(1)}=\left\{d_{1}^{*(1)}, d_{2}^{*(1)}, d_{3}^{*(1)}, d_{4}^{*(1)}\right\}=\{\langle 0.8,0,0.2\rangle,\langle 0.7,0,0.2\rangle,\langle 0.6,0.1,0.2\rangle,\langle 0.6,0,0.2\rangle\} \\
& d^{*(2)}=\left\{d_{1}^{*(2)}, d_{2}^{*(2)}, d_{3}^{*(2)}, d_{4}^{*(2)}\right\}=\{\langle 0.6,0.1,0.3\rangle,\langle 0.7,0.1,0.2\rangle,\langle 0.6,0.1,0.2\rangle,\langle 0.7,0,0.2\rangle\} \\
& d^{*(3)}=\left\{d_{1}^{*(3)}, d_{2}^{*(3)}, d_{3}^{*(3)}, d_{4}^{*(3)}\right\}=\{\langle 0.6,0.2,0.2\rangle,\langle 0.6,0,0.2\rangle,\langle 0.6,0.2,0.2\rangle,\langle 0.7,0.1,0.2\rangle\}
\end{aligned}
$$

Step 2: Calculate the GOWSNCS $\left(d_{i}^{(k)}, d^{*(k)}\right)$ by Equation (7) and obtain the collective cosine similarity matrix $V$ :

$$
V=\left[\begin{array}{ccc}
V_{1}^{1} & V_{1}^{2} & V_{1}^{3} \\
V_{2}^{1} & V_{2}^{2} & V_{2}^{3} \\
V_{3}^{1} & V_{3}^{2} & V_{3}^{3} \\
V_{4}^{1} & V_{4}^{2} & V_{4}^{3} \\
V_{5}^{1} & V_{5}^{2} & V_{5}^{3}
\end{array}\right]=\left[\begin{array}{ccc}
0.9411 & 0.9819 & 0.9817 \\
0.9967 & 0.9967 & 0.9938 \\
0.9796 & 0.9270 & 0.9471 \\
0.9864 & 0.9877 & 0.9758 \\
0.9415 & 0.9921 & 0.9921
\end{array}\right]
$$

Step 3: By Equation (8), we can obtain the collective overall value $R_{i}$ for the alternative $u_{i}(i=1,2$, $3,4,5)$ :

$R_{1}=0.9696, R_{2}=0.9958, R_{3}=0.9508, R_{4}=0.9836$, and $R_{5}=0.9770$

Step 4: Since $R_{2}>R_{4}>R_{5}>R_{1}>R_{3}$, the ranking order of the five alternatives is $u_{2}>u_{4}>u_{5}>u_{1}$ $>u_{3}$. which is the same as Ye's result (Ye 2014f). Thus, we can see that the alternative $u_{2}$ is the best choice among all alternatives.

Since the parameter value of $\lambda$ plays a role in the aggregation results, we take different values of $\lambda: 0.1,1,10,20, \ldots, 80$, to analyze how the different parameter values affect the ranking order of the alternatives. The ranking orders of the alternatives are shown in Table 1.

From Table 1, the ranking order of the alternatives is $u_{2}>u_{4}>u_{5}>u_{1}>u_{3}$ for $\lambda<50, u_{2}>u_{4}$ $=u_{5}>u_{1}>u_{3}$ for $\lambda=50$, and $u_{2}>u_{5}>u_{4}>u_{1}>u_{3}$ for $\lambda>50$. The best alternative is $u_{2}$ in all the ranking orders. Obviously, the different values of $\lambda$ in the GOWSNCS measure may affect the ranking 
Table 1. Ranking orders of the alternatives with the different parameter values of $\lambda$

\begin{tabular}{|l|l|}
\hline \multicolumn{1}{|c|}{$\lambda$} & \multicolumn{1}{c|}{$\quad$ Ranking Order of the Alternatives } \\
\hline 0.1 & $u_{2}>u_{4}>u_{5}>u_{1}>u_{3}$ \\
\hline 1 & $u_{2}>u_{4}>u_{5}>u_{1}>u_{3}$ \\
\hline 10 & $u_{2}>u_{4}>u_{5}>u_{1}>u_{3}$ \\
\hline 20 & $u_{2}>u_{4}>u_{5}>u_{1}>u_{3}$ \\
\hline 30 & $u_{2}>u_{4}>u_{5}>u_{1}>u_{3}$ \\
\hline 40 & $u_{2}>u_{4}>u_{5}>u_{1}>u_{3}$ \\
\hline 50 & $u_{2}>u_{4}=u_{5}>u_{1}>u_{3}$ \\
\hline 60 & $u_{2}>u_{5}>u_{4}>u_{1}>u_{3}$ \\
\hline 70 & $u_{2}>u_{5}>u_{4}>u_{1}>u_{3}$ \\
\hline 80 & $u_{2}>u_{5}>u_{4}>u_{1}>u_{3}$ \\
\hline
\end{tabular}

order of the alternatives. Therefore, the proposed decision making method along with taking different values of $\lambda$ corresponding to decision makers' preference and/or requirements is more flexible than other decision making methods based on similarity measures in the decision making process.

On the one hand, by comparison with the decision making methods (Zhou et al. 2014; Zheng et al. 2018, Lin et al. 2018, Liu et al. 2019a and 2019b), they cannot deal with decision making problems with indeterminate and inconsistent information in SNS setting. On the other hand, compared with the relative decision making methods based on similarity measures (Ye 2014d; 2014e; Ye 2017; Tu et al. 2018) and based on some aggregation operators (Ye 2014a; Zhang et al 2014; Liu and Wang 2014; Liu et al 2014; Yang and Li 2016; Sahin and Liu 2017) under simplified neutrosophic environments, the method proposed in this paper not only applies the GOWSNCS measure to group decision-making problems but also considers the weights of the ordered positions of cosine measure values. Thus, the proposed group decision making method with the parameterized family of the GOWSNCS measure makes it have more flexible and practical than the existing decision-making methods without the parameterized family (Ye 2014d; 2014e; Ye 2017; Sahin and Liu 2017; Tu et al. 2018). Furthermore, its aggregation calculation is simpler than the aggregation operators (Ye 2014a; Zhang et al 2014; Liu and Wang 2014; Liu et al 2014; Yang and Li 2016; Sahin and Liu 2017).

Obviously, the developed new method can alleviate the influence of unduly large or small similarities in the process of information aggregation due to considering the important degree of the cosine measure ordered positions and is simpler and more flexible than existing decision-making methods under SNS environment, which are the main advantages of the developed new method.

As the extension of the method introduced in (Zhou et al. 2014), the method proposed in this paper is different from the method in (Zhou et al 2014), and then their main differences are as follows:

- Our method uses the improved cosine similarity measure of SNSs based on the cosine function in the aggregation of similarity measures, while the method in (Zhou et al 2014) uses the cosine similarity measure of IFSs in vector space. Then, the improved cosine similarity measure is superior to the cosine similarity measure of IFSs in vector space since the former can overcome the disadvantages of the later in some cases (Ye 2014e) and the later cannot deal with the similarity measure of SNSs;

- The decision-making method in (Zhou et al 2014) only considers the associated weights for decision makers without considering the associated weights for attributes in the information aggregation, while our decision making method considers both; 
- Our group decision making method can handle decision-making problems with intuitionistic fuzzy information and simplified neutrosophic information, while the group decision-making method in (Zhou et al 2014) can only handle decision making problems with intuitionistic fuzzy information. Hence, our group decision-making method is superior to the group decision-making method in (Zhou et al 2014).

\section{CONCLUSION}

This paper proposed the GOWSNCS measure by combining the cosine similarity measure of SNSs with the GOWA operator and investigated its main properties and particular cases. The main advantages of the GOWSNCS measure are that it not only is a generalization of the cosine similarity measure but also considers the associated weights for attributes and decision makers in the information aggregation to alleviate the influence of unduly large or small similarities on the aggregation results by assigning them high or low weights. Then, a group decision making method based on the GOWSNCS measure was developed to handle multiple attribute group decision making problems with simplified neutrosophic information. Finally, an illustrative example was given to demonstrate the application and effectiveness of the proposed method.

Since the developed method can alleviate the influence of unduly large or small similarities in the process of information aggregation and is simpler and more flexible than existing decisionmaking methods under simplified neutrosophic environment, it provides a new way for solving multiple attribute group decision making problems with simplified neutrosophic information. Since there exist some measures/algorithms of SNSs, the GOWSNCS measure can be also extended to the aggregation of correlation coefficient, cross-entropy, and vector similarity measures for SNSs by the similar method. In future research, it is necessary and meaningful to continue working in the extension and application of the developed method to other domains, such as pattern recognition and medical diagnosis.

\section{ACKNOWLEDGMENT}

This paper was supported by the National Natural Science Foundation of China (No. 71471172).

\section{CONFLICT OF INTEREST STATEMENT}

I declare that I have no conflict of financial and personal relationships with other people or organizations. 


\section{REFERENCES}

Atanassov, K. (1986). Intuitionistic fuzzy sets. Fuzzy Sets and Systems, 20(1), 87-96. doi:10.1016/S01650114(86)80034-3

Atanassov, K., \& Gargov, G. (1989). Interval valued intuitionistic fuzzy sets. Fuzzy Sets and Systems, 31(3), 343-349. doi:10.1016/0165-0114(89)90205-4

Chi, P. P., \& Liu, P. D. (2013). An extended TOPSIS method for the multiple attribute decision making problems based on interval neutrosophic sets. Neutrosophic Sets and Systems, 1, 63-70.

Lin, Y., \& Wang, Y.M. (2018). Group decision making with consistency of intuitionistic fuzzy preference relations under uncertainty. IEEE/CAA Journal of Automation Sinica, 5(3), 741-748.

Liu, H. C., Wang, L. E., Li, Z. W., \& Hu, Y. P. (2019a). Improving risk evaluation in FMEA with cloud model and hierarchical TOPSIS method. IEEE Transactions on Fuzzy Systems, 27(1), 84-95. doi:10.1109/ TFUZZ.2018.2861719

Liu, H. C., Yang, M. Y., Zhou, M. C., \& Tian, G. D. (2019b). An integrated multi-criteria decision making approach to location planning of electric vehicle charging stations. IEEE Transactions on Intelligent Transportation Systems, 20(1), 362-373. doi:10.1109/TITS.2018.2815680

Liu, P. D., Chu, Y. C., Li, Y. W., \& Chen, Y. B. (2014). Some generalized neutrosophic number Hamacher aggregation operators and their application to group decision making. International Journal of Fuzzy Systems, 16(2), 242-255.

Liu, P. D., \& Wang, Y. M. (2014). Multiple attribute decision-making method based on single-valued neutrosophic normalized weighted Bonferroni mean. Neural Computing \& Applications, 25(7-8), 2001-2010. doi:10.1007/ s00521-014-1688-8

Peng, J. J., Wang, J. Q., Zhang, H. Y., \& Chen, X. H. (2014). An outranking approach for multi-criteria decisionmaking problems with simplified neutrosophic sets. Applied Soft Computing, 25, 336-346. doi:10.1016/j. asoc.2014.08.070

Sahin, R., \& Liu, P. (2017). Possibility-induced simplified neutrosophic aggregation operators and their application to multi-criteria group decision-making. Journal of Experimental \& Theoretical Artificial Intelligence, 29(4), 769-785. doi:10.1080/0952813X.2016.1259266

Smarandache, F. (1999). A unifying field in logics. Neutrosophy: neutrosophic probability, set and logic. Rehoboth: American Research Press.

Tu, A., Ye, J., \& Wang, B. (2018). Symmetry measures of simplified neutrosophic sets for multiple attribute decision-making problems. Symmetry, 10(5), 144. doi:10.3390/sym10050144

Wang, H., Smarandache, F., Zhang, Y. Q., \& Sunderraman, R. (2005). Interval neutrosophic sets and logic: theory and applications in computing. Phoenix, AZ: Hexis.

Wang, H., Smarandache, F., Zhang, Y. Q., \& Sunderraman, R. (2010). Single valued neutrosophic sets. Multispace and Multistructure, 4, 410-413.

Yager, R. R. (2004). Generalized OWA aggregation operators. Fuzzy Optimization and Decision Making, 3(1), 93-107. doi:10.1023/B:FODM.0000013074.68765.97

Yang, L., \& Li, B. (2016). A multi-criteria decision-making method using power aggregation operators for single-valued neutrosophic sets. International Journal of Database Theory and Appllications, 9(1), 23-32. doi:10.14257/ijdta.2016.9.2.04

Ye, J. (2013). Multicriteria decision-making method using the correlation coefficient under single-valued neutrosophic environment. International Journal of General Systems, 42(4), 386-394. doi:10.1080/0308107 9.2012.761609

Ye, J. (2014a). A multicriteria decision-making method using aggregation operators for simplified neutrosophic sets. Journal of Intelligent \& Fuzzy Systems, 26(5), 2459-2466. 
Ye, J. (2014b). Single valued neutrosophic cross-entropy for multicriteria decision making problems. Applied Mathematical Modelling, 38(3), 1170-1175. doi:10.1016/j.apm.2013.07.020

Ye, J. (2014c). Similarity measures between interval neutrosophic sets and their applications in multicriteria decision-making. Journal of Intelligent \& Fuzzy Systems, 26, 165-172.

Ye, J. (2014d). Multiple attribute group decision-making method with completely unknown weights based on similarity measures under single valued neutrosophic environment. Journal of Intelligent \& Fuzzy Systems, 27(12), 2927-2935.

Ye, J. (2014e). Vector similarity measures of simplified neutrosophic sets and their application in multicriteria decision making. International Journal of Fuzzy Systems, 16(2), 204-211.

Ye, J. (2015). Improved cosine similarity measures of simplified neutrosophic sets for medical diagnoses. Artificial Intelligence in Medicine, 63(3), 171-179. doi:10.1016/j.artmed.2014.12.007 PMID:25704111

Ye, J. (2017). Simplified neutrosophic harmonic averaging projection-based method for multiple attribute decision making problems. International Journal of Machine Learning and Cybernetics, 8(3), 981-987. doi:10.1007/ s13042-015-0456-0

Zadeh, L. A. (1965). Fuzzy sets. Information and Control, 8(3), 338-353. doi:10.1016/S0019-9958(65)90241-X

Zhang, H. Y., Wang, J., \& Chen, X. H. (2016). An outranking approach for multi-criteria decision-making problems with interval-valued neutrosophic sets. Neural Computing \& Applications, 27(3), 615-627. doi:10.1007/ s00521-015-1882-3

Zhang, H. Y., Wang, J. Q., \& Chen, X. H. (2014). Interval neutrosophic sets and their application in multicriteria decision making problems. The Science World Journal. doi:10.1155/2014/645953

Zheng, J., Wang, Y.M., \& Chen, S.Q. (2018). Dynamic case retrieval method with subjective preferences and objective information for emergency decision making. IEEE/CAA Journal of Automation Sinica, 5(3), $749-757$.

Zhou, L., Tao, Z., Chen, H., \& Liu, J. (2014). Intuitionistic fuzzy ordered weighted cosine similarity measure. Group Decision and Negotiation, 23(4), 879-900. doi:10.1007/s10726-013-9359-1

Jun Ye graduated and received his M.S. degree in Automation and Robotics from the Technical University of Koszalin, Poland in 1997. From Feb. to Aug. 2012, he was a visiting scholar in the School of Engineering of Southern Polytechnic State University in USA. Now, he is a professor in the Department of Electrical and Information Engineering, Shaoxing University, China. He has more than 30 years of experience in teaching and research. His research interests include soft computing, multi-criteria decision-making, robotics, intelligent control, pattern recognitions, and fault diagnosis. He has published more than one hundred papers in journals. He has written few books related to his research work. He has finished a few projects sponsored by government of China. 